\title{
Vivid memories of emotional events: The accuracy of remembered minutiae
}

\author{
FRIDERIKE HEUER and DANIEL REISBERG \\ Reed College, Portland, Oregon
}

\begin{abstract}
It has been claimed that emotional arousal causes a narrowing of attention, and, therefore, impoverished memory encoding. On this view, if details of an emotional event are reported subsequently, these details must be after-the-fact reconstructions that are open to error. Our study challenges these claims. Using a long-term (2-week), incidental learning procedure, we found that emotion promotes memory both for information central to an event and for peripheral detail. This contrasts with the results of explicit instructions to remember or to attend closely to the event, both of which seem to promote memory for the event's gist at the expense of detail. The likely mechanisms underlying these effects are discussed.
\end{abstract}

A number of studies have indicated that the emotionality of an event is an excellent predictor for how vividly the event is recalled. The extreme case consists of "flashbulb memories," which are extremely vivid and detailed memories of very emotional events (Brown \& Kulik, 1977; Pillemer, 1984; Reisberg, Heuer, McLean, \& O’Shaughnessy, 1988; Rubin \& Kozin, 1984). Not only are emotional events recalled with much detail, but the detail is of a peculiar sort: We often remember seemingly trivial aspects of emotional events, including many incidental sights and sounds that accompanied the event, the particular way that sentences were phrased, and so forth.

How accurate are these memories? In addition to being theoretically important, this question is also pertinent to the debate concerning the reliability of eyewitness memory. That is, eyewitnesses to crimes are often emotionally aroused, since crimes tend to be frightening or upsetting. In addition, eyewitnesses are often asked, after the crime, to remember minute details of how things unfolded.

The research literature makes an unambiguous claim about this conjunction of emotionality and detail memory. The claim is that emotional arousal causes a narrowing of one's attention and, hence, inattention to a great many peripheral details. For eyewitnesses, this results in the so-called "weapon focus" (see Loftus, 1979). In the laboratory data, the analogous claim is generally referred to as the Easterbrook hypothesis (e.g., see Bruner, Matter, \& Papanek, 1955; Easterbrook, 1959; Loftus, 1982; Mandler, 1975).

\footnotetext{
The experiment reported here was completed as part of Friderike Heuer's doctoral dissertation. We thank Jerome Bruner, Joseph Greenbaum, and Arien Mack for their comments on all aspects of this research, and Reed College for providing facilities. Address correspondence to either author at Reed College, Psychology Department, Portland, Oregon 97202 .
}

If emotion leads in this way to the exclusion of information, then it should create relatively impoverished memories of the peripheral information in an event, but it ought to enhance memories of the central information (i.e., nothing but the gun would be remembered, but the gun would be remembered with great clarity). On this view, if peripheral details are reported subsequently (as they often are), it is not because they have been accurately remembered; they must instead be after-the-fact reconstructions that therefore are open to error.

Note the ironic contrast here: Emotion is claimed to lead to vivid recollections, but to impoverished encoding. This implies that it is our most detailed memories, memories in which we are likely to be most confident, that are also most likely to be filled with reconstructed, and potentially inaccurate, detail. Indeed, several authors, notably Loftus (1979, 1982), Neisser (1982; but see also Neisser, 1986; Thompson \& Cowan, 1986), and McCloskey, Wible, and Cohen (1988), have suggested that even these very vivid recollections are often inaccurate.

Unfortunately, the evidence for this chain of claims is for the most part indirect. As McCloskey and Egeth (1983) point out, much of the evidence for the "weapon focus" is anecdotal. Evidence for the Easterbrook claim comes largely from animal studies, with tasks or situations that differ from those facing the eyewitness. As we will discuss below, one such difference seems critical: Eyewitnesses are typically called on to remember the event some hours (or days) after the occurrence; the Easterbrook data typically focus on animals' immediate use of various stimulus cues. Even in the few relevant studies with humans (e.g., Loftus \& Burns, 1982), the retention interval has been quite short (10-15 $\mathrm{min}$; for a recent review of this literature, see Eysenck, 1982).

In several studies, researchers have looked at arousal's effects on retention and reported that arousal improves the accuracy of long-term retention. (See, e.g., Clark, Milberg, \& Ross, 1983; Kleinsmith \& Kaplan, 1963.) Unfortunately, for our purposes, in these studies word lists 
or stories have often been used as the to-be-remembered materials. Consequently, the studies cannot easily speak to the question of emotion's impact on memory for more peripheral information, which is critical to the weaponfocus or Easterbrook claims.

A recent group of studies by Christianson and Loftus does pertain more directly to the accuracy of remembered minutiae, but these studies still leave the issue unsettled. Christianson and Loftus (1987) examined subjects' memories of either neutral or traumatic events, portrayed in a series of slides, and reported data consistent with the Easterbrook claim: enhanced memory for central materials and poorer memory for peripheral detail. However, the subjects in this study were explicitly given a "focus" task-they were instructed to write down the most distinguishing feature in each slide shown. This task seems likely to create a selective set which may itself interact with arousal and thus renders the study, we believe, a poor analogue to the eyewitness's situation.'

This manipulation of selective set is absent from studies by Christianson (1984) and Christianson, Loftus, and Nilsson (1987), but the "detail" memory examined in those studies was defined in a manner different from that intended here. The Christianson et al. (1987) study also yielded data supporting the Easterbrook claim: Arousal improved retention of central information, but diminished retention of peripheral detail. However, "central" information was defined in a fashion that included any detail pertinent to central characters in the plot. (That is, if a slide showed, as its central character, a woman riding a bicycle, the color of the woman's coat would be considered central.) This data analysis therefore blurs the distinction between memory for the gist or schema of a story and memory for details of appearance and manner.

Finally, Christianson (1984) had subjects watch a series of slides that depicted a story. In one condition, the story was neutral in content; in the other condition, the story contained traumatic material (a graphically depicted car accident). Detail memory was assessed in a recognition test in which subjects chose which pictures they had seen as part of the series, with the distractor choices differing from the original only in camera angle. The results showed that "forgetting rates from initial to delayed tests for the traumatic groups (were) significantly (lower) from those of the neutral group"' (Christianson, 1984, p. 157). In some cells of the design, this produced a crossover, with an arousal disadvantage in immediate testing but an advantage with delay. But this pattern was not consistent across the design.

We wish to suggest, however, that memory for camera angle is an overly stringent assessment of detail memory. More importantly, camera angle seems a far cry from the kinds of details which fill our vivid recollections. If we are to assess the accuracy of the kinds of details in, say, a "flashbulb" memory or an eyewitness's testimony, we will need more appropriately chosen tests. With these considerations in mind, we modeled the present study after the Christianson (1984) design, in order to assess the impact of arousal on detail memory.

In the present study, subjects watched a sequence of slides accompanied by a taped narration. In the neutral version, the subjects heard and saw a story about a boy and his mother going to visit his father at work. In the story, the father is chief mechanic at a local garage. He successfully repairs a car, and the mother leaves, stopping to telephone her boss to announce that she will be late for her own work.

In the arousal version of this story, the mother and son again visit the father at work, but this time the father is chief surgeon at a local hospital. He successfully performs surgery, and the middle slides of the series show both the surgery itself and also the patient's badly damaged legs. The mother leaves the hospital, again stopping to telephone her boss to announce that she will be late. These two stories were matched as much as possible for structure, style, layout of the slides, and so forth.

Because we wanted to approximate as closely as possible the circumstances of learning outside the laboratory, the subjects had no expectation of a later memory test, and they had no explicit task at the time of learning. As in Christianson's designs, the subjects were simply told that we were studying physiological reactions to different kinds of materials. When the subjects returned to the laboratory 2 weeks later, expecting to see a different presentation, they were instead given a surprise memory test. This cover story also provided an excuse to monitor the subjects' heart-rates, so that we could make sure that our arousal manipulation was successful.

Two other groups were also included, so that we could examine the mechanisms behind the effects of arousal. For direct assessment of the effects of attentiveness, the subjects in the problem-solving group watched the neutral materials, but were told that the materials were designed to mimic some recent event in the news or a popular movie. Their task was to discern what event was being mimicked. Of course, there was no such event, and, 2 weeks later, they too received a memory test. Finally, the subjects in the memorizing group also watched the neutral material, but they were simply told at the outset that their memories would be tested, and that they should remember as much of the story and as many of its details as they could.

Two types of memory test were included, recall and recognition, and memory was assessed for two kinds of material, elements central to the story that had been presented 2 weeks prior, and details peripheral to the story. The recall measure was used to examine both the content and the form of the subjects' spontaneous recollections; the forced-choice recognition measure was used to assess memory in a more fine-grained way, less influenced by patterns of report bias.

The contrast between central and peripheral information is critical for assessing the claim that emotion narrows the range of attended materials, and hence of what 
will be remembered. If the Easterbrook hypothesis is applied to this procedure, the arousal group should yield worse memory for peripheral information than the neutral group, although the arousal group is likely to have better retention of central materials. The memorizing group should resemble the arousal group in both these regards, given the many demonstrations of "gistification" or "schematization" in memory (see, e.g., Bartlett, 1932; Minsky, 1975; and many of the studies in Rubin, 1986.) Finally, it would seem likely that the problem-solving instructions would lead subjects to spread their attention more broadly, and so one would expect better memory for peripheral materials in this group. However, none of these predictions turned out to be correct.

\section{METHOD}

\section{Subjects}

Subjects were 40 undergraduates from Reed College, ranging in age from 19 to 24 years. Although they received no direct payment, participation in the study entered them in a lottery for a $\$ 50$ prize. The subjects were randomly assigned to one of four groups, with the constraint that each group contain 5 women and 5 men.

The subjects were instructed that they were to return to the laboratory for a second session after a 2-week interval. Those who failed to return ( 1 in the neutral, 2 in the problem-solving group) were replaced from the same pool.

\section{Stimuli and Apparatus}

Two sets of story materials were prepared. Each consisted of 12 slides, with each slide accompanied by a simple taped sentence. The slides were projected onto a rear-projection screen at a size of $20 \times 14 \mathrm{in}$. The subjects sat $31 \mathrm{in}$. from the screen. The taped sentences were heard from a speaker situated behind the screen. Each slide was shown for $6 \mathrm{sec}$, with a $1-\mathrm{sec}$ interstimulus interval. The corresponding sentence began approximately $500 \mathrm{msec}$ after the slide was displayed, and ended approximately $4 \mathrm{sec}$ later.

To assess the subjects' arousal, heart rate was monitored by means of a CIC heart-rate speedometer, with a photosensitive cell clipped to the subject's earlobe while the head was stabilized in a headrest. The monitor gave a digital output that was recorded by the experimenter for baseline rates and during the slide presentation. Both the experimenter and all the equipment were out of sight of the subject during the procedure.

The slides depicted a simple story. In both versions, a mother and her son visit the father at his workplace and see him perform a typical task. The mother then departs late for work, makes a telephone call, and hails a cab. The neutral version shows the father as a garage mechanic fixing a car shown previously as broken down. The arousal version depicts the father as a surgeon operating on a victim of an accident shown previously. Both the visual and the auditory aspects of these stories were matched as much as possible, and both versions had the same initial three and final four slides. (The actual sentences used, for both the arousal and the neutral versions of the story, are listed in the Appendix.)

For both groups, Slide 4 depicted "father's workplace." For the arousal subjects, the slide showed a building clearly labelled "hospital"'; for the other subjects, the slide showed a garage. Slide 5 for the arousal subjects showed an accident scene; for the other subjects, Slide 5 showed the broken car. The material designed to arouse the subjects depicted a team of surgeons bending over a patient whose viscera were plainly visible (Slide 6); Slide 7 showed the badly scarred legs of a child, and Slide 8 presented the father removing his surgical gloves (one of two black and white pictures mixed into the other colored slides). The corresponding slides for the other groups showed a team of mechanics bending over an engine; a broken car part; and the father washing his greasy hands.

\section{Procedure}

The subjects were run in individual sessions and told that we were interested in the patterns of physiological reactions to different types of visual stimulation. They expected to watch different types of material, "some of which might appear to be either pleasant, unpleasant or erotically tinged," in two sessions separated by 2 weeks.

The subjects were seated, and the photocell was fastened to their earlobes. Then they were instructed in the procedure, assured that they could leave the experiment at any time, and given an opportunity to ask questions for clarification. The instructions and set-up spanned about $15 \mathrm{~min}$, after which the first baseline measurements were taken. Baseline heart-rate measurements were recorded over a 2-min period; the reported baselines are the mean heart rate during this period. At the end of the baseline measurement, the presentation began.

The arousal group watched the version of the story with the hospital scenes at its center. They were instructed only to attend to the story while their heart rates were monitored. The remaining three groups viewed the version with the garage scenes at its center. The neutral group received the same instructions as did the arousal group. The memorizing group was additionally instructed to "memorize as much as possible of both the central storyline of the story ... as well as all of the circumstantial details." The problem-solving group was instructed that the study also involved "the relationship between physiological reactions and problem solving." They were told that one of the stories they would see was "modeled after a recent event in the news or a popular movie. We have deliberately changed some things in order to disguise what the real event is. But enough of the original event, both with regard to the storyline and circumstantial details, is unchanged. Your task is to discover what the original is. But remember it might be this story or the next."

After the slide presentation was over, the subjects were asked to rate on a 9-point scale (from very strong agreement to very strong disagreement) how emotional they thought the story was and how emotionally they had reacted to it. They were then given the questionnaire measure of stimulus screening and arousability (Mehrabian, 1977). This is a questionnaire that asks about sensitivity to external stimulation regarding noises, smells, perceptual events, kinesthetic experiences, and so forth. The subjects took this questionnaire home under instructions to fill it out and bring it back at their second visit (38 out of 40 subjects complied with this request). This procedure was intended to persuade the subjects that we were truly interested in their reactions to different types of stimulation inside and outside of the laboratory.

In the second session, 2 weeks later, the subjects were told that there would be no second story. Instead they were asked to recall as much as they could of the first presentation. They were urged to recall "the story as much as any particular detail which you remember having seen or heard about." The subjects were told to report what they remembered in whatever sequence the material came to mind, and they were given as much time as they needed to complete their reports.

After the subjects indicated that they could remember no more, the vividness of visual imagery questionnaire (Marks, 1972) was administered. This questionnaire allowed us to separate briefly the recall and recognition measures. The subjects' recognition was then assessed in a series of 120 four-alternative forced-choice (4AFC) questions ( 10 per slide), comprising 78 peripheral and 42 central items. The subjects expressed a confidence rating for each answer, using a 5-point scale ranging from "absolutely certain" to "totally guessing." All subjects received the questions in the order of the original slide presentation and in the same sequence within any given slide. (This was necessary, since some questions presupposed others.) 
Finally, recognition memory was assessed for the 12 sentences heard concurrently with the slides. Each sentence was visually presented among three distractor sentences (approximate paraphrases), which were matched in length, syntax, and so forth, as much as possible. The subjects' narrations, their answers to the recognition items, and their confidence ratings were tape-recorded for later analysis.

Four independent judges had categorized each recognition item as assessing either central or peripheral information. The judges were instructed to consider as central any fact or element pertaining to the "basic story" that could not be "changed or excluded without changing the basic story line." The instructions to the judges borrowed language from Rosch's (1978) notion of a "basic level" of description, so that judges regarded as peripheral detail anything below this basic level. Thus, for example, a description of a slide as showing "a woman crossing an intersection" would be considered basic. To describe the same scene as "a person outside" would be too general, while "a red-haired woman with a handbag on her left arm stepping onto the first third of the cross walk" would be regarded as too specific. The judges agreed on almost all items; in cases of disagreement, items were replaced. Some sample peripheral items were: the color of the door in front of which the mother stood in a particular slide; whether the boy was carrying a lunchbox or a soccer ball; and so forth. Some sample central items were: whether the mother and son were going to the gas station, to school, or shopping; whether the son watched the repair, or played in the back room; and so forth. Not surprisingly, a larger number of items were judged as peripheral ( 78 of 120 ), since presumably the peripheral details surround the thematic chunks in large number.

\section{RESULTS}

\section{Arousal Data}

Baseline heart-rate levels were taken in the 2-min interval before the slide presentation began. A one-way ANOVA (four levels) showed no reliable differences among the groups' baseline levels $[F(3,36)<1.0]$. Our subjects varied considerably in their baseline heart rates, with rates ranging from 54 to 103 beats per minute. However, the four group means are all within 2.5 beats per minute of each other (see Figure 1).

During the slide presentation, an average heart rate was recorded for each subject during each slide. These were then averaged to yield values for each of the three phases of the presentation: during Slides 1-3 (which were the same for all groups), Slides 4-8 (which were different for the arousal subjects), and then Slides 9-12 (again, the same for all groups). A $4 \times 3$ ANOVA (group $\times$ phase) showed no main effect of group $[F(3,36)<1.0]$, but a reliable effect of phase $[F(2,72)=4.553, p<.02]$ and, critically, a group $\times$ phase interaction $[F(6,72)=3.355$, $p<.01]$.

As can be seen in Figure 1, the subjects in the arousal group showed a striking drop in heart rate across the phases, a pattern clearly different from that shown by the other groups. Post hoc tests confirm this pattern: The arousal group by itself showed a significant effect of phase $[F(2,72)=10.639, p<.005] ;$ no other group showed an effect of phase.

We note that Figure 1 is misleading in one regard: The four groups appear to differ in Phase 1, during which they viewed the exact same slides and heard the same sentences. However, this apparent difference is not statistically reliable. An analysis of just the Phase 1 data shows no significant difference among the groups $[F(3,38)<1.0]$.

In Figure 1, the arousal group does not show the heartrate acceleration one would associate with a defensive

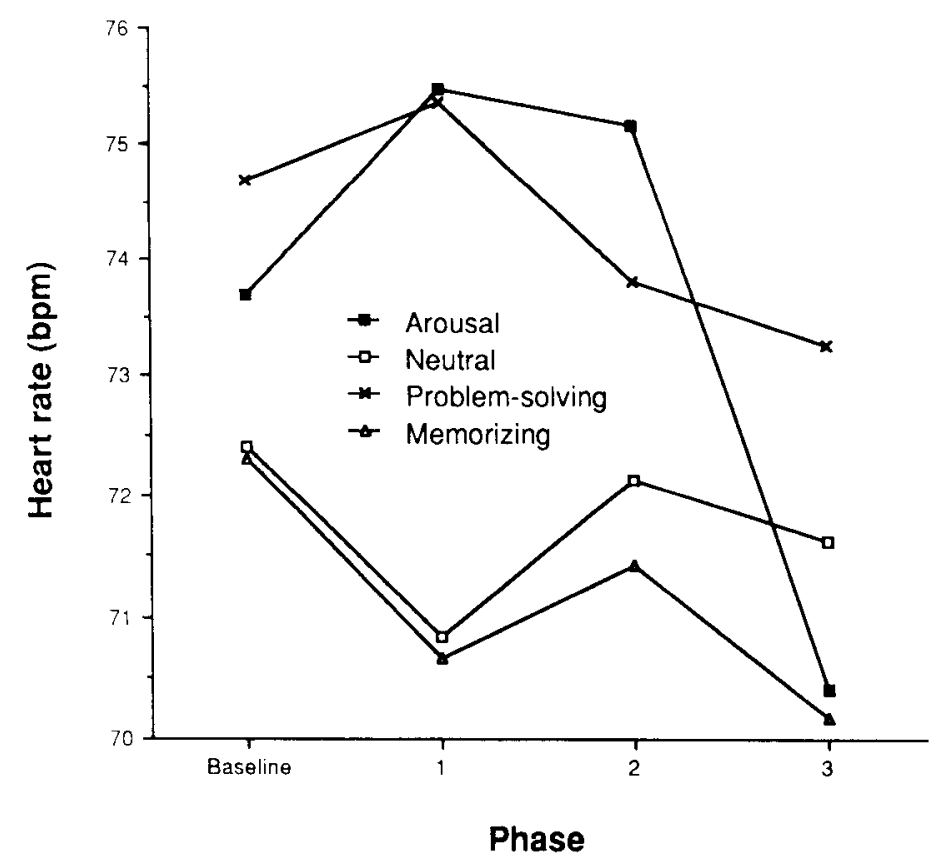

Figure 1. Baseline levels and changes in heart rate, summarized by phase of the procedure, in the arousal, neutral, problem-solving, and memorizing groups. 
reaction toward noxious stimuli. However, the literature identifies two distinct heart-rate patterns that characterize arousal. In addition to the acceleration pattern, Lacey and Lacey (1974) argue that some stimulation evokes an orienting response, which, in turn, is linked to a feedback mechanism through which cardiac deceleration increases cortical arousal. Likewise, Bohlin and Graham (1977) argue that heart-rate deceleration occurs with the specific type of arousal associated with an orienting response (see also Graham, 1979, pp. 137-167). We note in passing that this heart-rate deceleration is not unique to our study, but has also appeared in other procedures designed to test the Easterbrook claim (e.g., see Christianson, 1984). We note in addition that the animal studies cited in support of the Easterbrook claim also presumably involve this "orienting" type of arousal, inasmuch as the arousal stimulus in these studies is typically food for food-deprived animals.

The arousal effect was also evident in the subjects' selfreports. In judging the emotionality of the story itself, on a scale ranging from -4 to +4 , the arousal subjects rated the presentation at 2.3 , the neutral subjects at -1.7 , the memorizing group at -1.9 , and the problem-solving group at 0.5 . A one-way ANOVA showed this difference to be highly reliable $[F(3,36)=12.336, p<.001]$. Similar ratings were obtained for the question about how much the subjects themselves had been moved by the presentation, with ratings of 1.3 from the arousal subjects, and $-1.9,-2.9$, and -1.4 for the other three groups, respectively $[F(3,36)=8.78, p<.001]$.

\section{Recall Data}

The recall protocols were scored partially blindly as to which group was being scored. (The recall protocols for the arousal story were obviously distinguishable; those for the other three groups were not.) The protocols were coded into central and peripheral items recalled, using the same criteria that the judges used in categorizing the recognition items. (This was appreciably eased by the considerable overlap between the pool of items recalled and the pool of items used in the recognition, particularly for the central category.)

Table 1 shows the mean amount correctly recalled by each of the four groups. Although the table shows a consistent advantage for the arousal group in recall, this difference is not statistically reliable for either total information recalled or the peripheral recall. Looking at the total recall first, the strongest of the contrasts is that between the arousal group's performance and that of the neutral group $[t(17)=1.38$, n.s. $] .{ }^{2}$ In peripheral recall, the strongest of the contrasts is between the arousal group and the problem-solving group $[t(17)=1.78$, n.s.]. However, the arousal subjects' advantage is statistically reliable for recall of central information. The contrast between the arousal group and the neutral group yields a $t(17)=2.34, p<.05$; the contrast between the memorizing and neutral groups is also significant $[t(17)=2.11$,
Table 1 Mean Number of Correctly Recalled Items

\begin{tabular}{|c|c|c|c|c|}
\hline Group & $\begin{array}{c}\text { Central } \\
\text { information }\end{array}$ & $\begin{array}{l}\text { Peripheral } \\
\text { information in }\end{array}$ & $\begin{array}{l}\text { Total } \\
\text { fformation }\end{array}$ & $\begin{array}{c}\text { Number of } \\
\text { Slides Recalled }\end{array}$ \\
\hline Arousal & 10.60 & 12.50 & 23.10 & 7.31 \\
\hline Neutral & 6.44 & 8.56 & 15.00 & 4.67 \\
\hline Problem solving & 8.11 & 6.40 & 14.33 & 5.56 \\
\hline Memorizing & 8.70 & 9.11 & 17.70 & 7.20 \\
\hline
\end{tabular}

$p<.05]$. None of the other contrasts reached significance levels.

The fact that recall levels are.higher in this table for peripheral information should not be surprising. In the presented stories, the quantity of reportable peripheral information far exceeds that of central information. If the absolute numbers shown in Table 1 could be converted to "proportions," the apparent advantage of peripheral recall would likely be reversed. We did not attempt such a transformation, because there was no baseline against which to compute proportions (i.e., no count of all the reportable details).

The subjects' narrations were also scored for which of the slides and accompanying sentences were reported. The subjects were given credit for remembering a slide if anything visually contained in that slide appeared in their narrations. Similarly, any recall that could only have come from a particular sentence was sufficient for credit for remembering the sentence. The arousal group recalled the largest number of slides, the neutral subjects the least. The difference between these two extreme groups is reliable at the $p<.01$ level $[t(17)=2.86]$, as is the contrast between the arousal group and the problem-solving group $[t(17)=2.02, p<.05]$.

\section{Recognition Data}

The results of the $4 \mathrm{AFC}$ test were analyzed with a 4 $\times 2 \times 3$ ANOVA, with the factors of group, central versus peripheral information, and phase of the story presentation (using the same partitions as in the heart-rate analysis). The ANOVA showed a significant effect of phase $[F(2,72)=6.540, p<.01]$, with best performance in the middle phase. There was also a tendency toward better memory for peripheral materials than for central materials $[F(1,36)=3.203, p<.10]$. The factors of phase and central/peripheral information strongly interacted $[F(2,72)=49.266, p<.001]$ : Memory for central materials was at its best in the final phase of the presentation, at its worst in the first phase. Memory for peripheral materials showed essentially the reverse pattern-worst in the final phase, as compared with the two earlier phases.

The critical results, however, concern the contrasts among the four groups. The ANOVA showed a reliable main effect of group $[F(3,36)=3.217, p<.05]$. Best performance was observed in the arousal group and worst in the neutral group, with the other two groups falling in between. Not surprisingly, there was also a strong 
group $\times$ phase interaction $[F(6,72)=2.28, p<.05]$. The arousal group showed a robust effect of phase $[F(2,72)=6.838, p<.005]$; no other group showed this effect. [The memorizing group showed a trend toward a phase effect, with $F(2,72)=2.462, p<.10$.] As Figure 2 indicates, the arousal group's advantage was most pronounced in the middle phase of the procedure; in the final phase, the arousal group's performance was matched by that of the problem-solving group.

Are the memory benefits of arousal evident for both peripheral and central memory? The ANOVA showed no hint of an interaction between the group effect and type of material $[F(3,36)=.382, p>.50]$, nor was there a group $\times$ phase $\times$ central/peripheral information interaction $[F(6,72)=.934, p>.25]$. As a different way of making this point, $t$ tests were used to compare the arousal and neutral groups for each phase of the procedure, separately for peripheral and for central memory. Not surprisingly, there was no contrast between these groups in remembering the presentations' first phase, prior to the onset of arousal [for central materials, $t(18)=.775$; for peripheral materials, $t(18)=.100$ ]. In the procedure's middle phase, the arousal group outperformed the neutral group with both central and peripheral materials $[t(18)=3.298, p<.005$, and $t(18)=3.104, p<.01$, respectively]. In the procedure's final phase, arousal subjects still tended to have an advantage with peripheral materials $[t(18)=1.868, p<.08]$, but they had no advantage in remembering central materials.

This phase-by-phase analysis also lets us explore the performance of the problem-solving and memorizing groups. Figure 2 indicates that these groups performed at levels intermediate between those of the arousal and neutral groups; the statistics bear this out. Performance tended to be better in the memorizing and problem-solving groups than in the neutral group, but this contrast never reached statistical significance. The two strongest contrasts are in memory for central materials: The memorizing group tended to outperform the neutral group in the procedure's middle phase $[t(18)=1.783, p<.10]$; the problem-solving group tended to outperform the neutral group in the final phase $[t(18)=1.789, p<.10]$.

Similarly, the problem-solving and memorizing groups tended to perform less well than the arousal group. This contrast was statistically reliable for the problem-solving group's memory for central materials from the procedure's middle phase $[t(18)=3.200, p<.01]$. Likewise, the arousal subjects outperformed the memorizing group in memory for peripheral materials from the procedure's final phase $[t(18)=2.783, p<.05]$. No other contrasts reached statistical significance.

Finally, Figures 3 and 4 allow a close examination of arousal's impact on central and peripheral performance. These figures break the data down according to the slide within the sequence being probed by each recognition question: Figure 3 shows performance for central information; Figure 4 shows peripheral information. In Figure 3, we can see that all groups remember the central information from the second half of the presentation better. Note the clearly parallel data from the four groups, indicating that we succeeded in designing stories matched in complexity, structure, and so on.

Of particular interest, though, is Figure 4. Arousal subjects showed no particular advantage in remembering

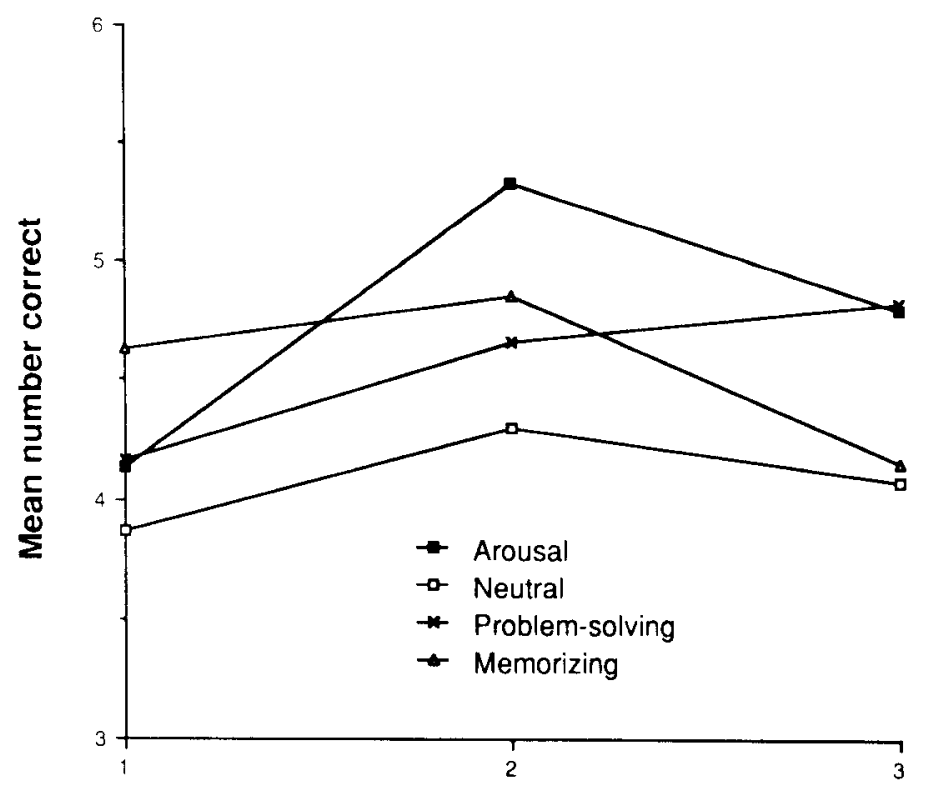

Phase

Figure 2. Recognition performance for all four groups. 


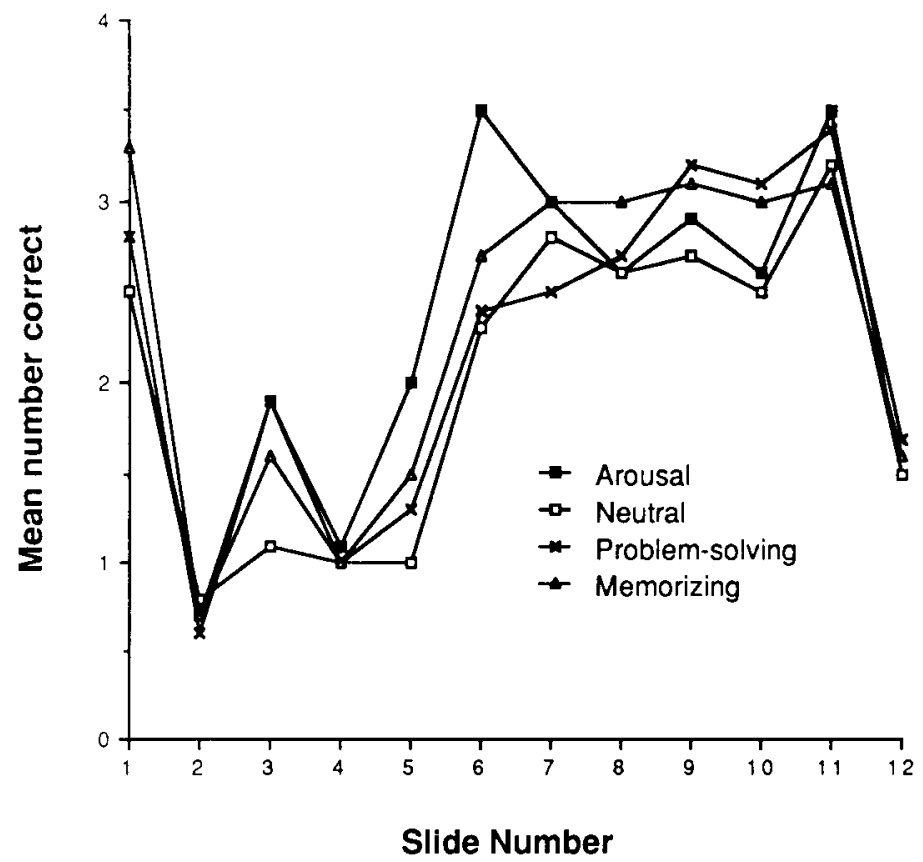

Figure 3. Slide-by-slide recognition performance for all four groups for central information.

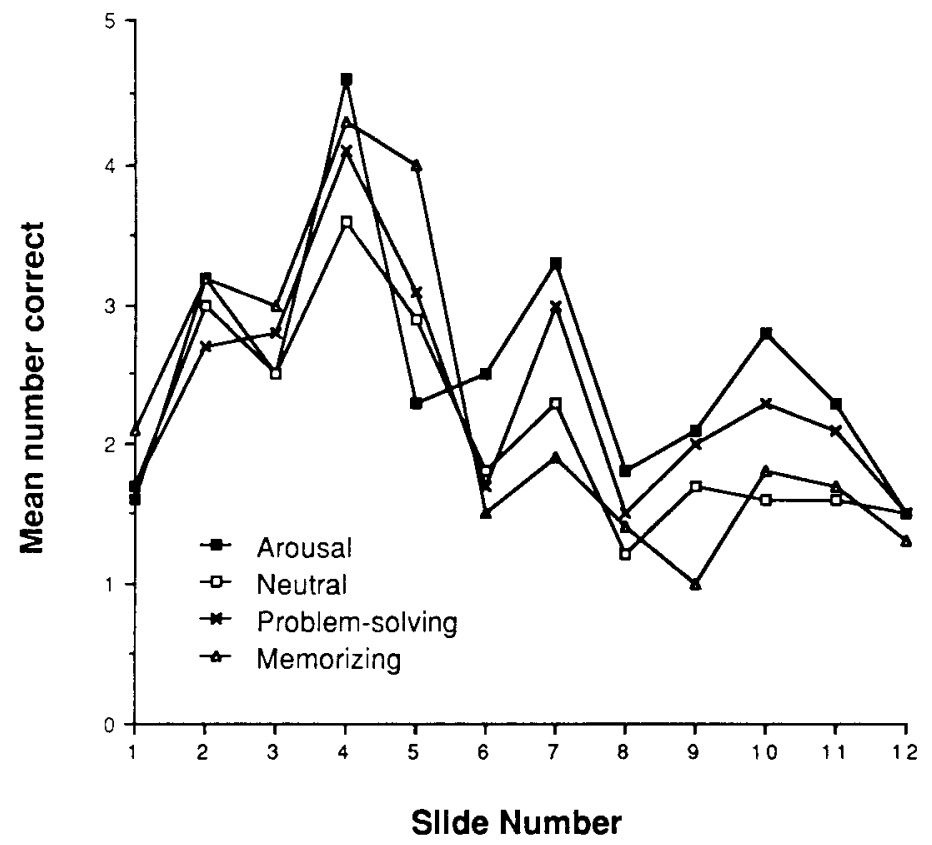

Figure 4. Slide-by-slide recognition performance for all four groups for peripheral information. 
peripheral details from the first half of the presentation (Slides 1-5)-that is, prior to the onset of arousal. Arousal subjects did, however, have a clear advantage in remembering details from all the slides subsequent to the arousal's onset, including the final three slides, which were, of course, identical for all four groups. The advantage is not huge, but it is rather consistent. This pattern shows up as the statistically reliable group $\times$ phase interaction.

It is interesting also to compare Figure 4 with Figure 5, which shows the slide-by-slide cardiac values. The distinct downward turn in the arousal group begins with Slide 6 (the first slide in which the memory advantage appears). Apparently, then, the data indicate that the memory advantage and the physiological effect go handin-hand.

\section{Intrusion Errors}

So far, the data indicate that arousal promotes retention both of central information and of peripheral details. At the same time, a different aspect of our data indicates that emotion does not uniformly lead to accurate memories.

Our early analyses of the recall data looked only at how many true propositions were contained in the protocols; in this regard, the recall data provide a muted echo of the recognition findings, with a memory advantage for the arousal subjects. However, our reading of the recall protocols suggested to us that arousal and neutral subjects made rather different sorts of intrusion errors. Roughly speaking, subjects in the neutral group tended to err about the plot, often importing events that had not appeared at all. For example, a subject might remember that the mother brought the child to school, or that the mother went grocery shopping after she left the garage, and so on. In general, these subjects seemed to be constructing sensible but false aspects of the tale, as if to fill the gaps in the remembered sequence.

In contrast, arousal subjects made very few plot errors. Instead, these subjects tended to confabulate about the protagonists' motives or reactions or expressed feelings, often exaggerating what the story had said about these, sometimes falsifying what the story had said. For example, one subject reported vivid recollections of hearing that the mother was upset that the child saw the operation; another that she was angry that the father allowed the son to watch the surgery, although there was no mention of these in the story. To put this in Bartlettian terms, these subjects seem to have remembered an "attitude," which they struggled in their recollections to justify.

To test these impressions, subjects' intrusion errors were coded into two (post hoc) categories - "plot" errors, and "character" errors. ${ }^{3}$ The former category included reports of actions or locations that had not been mentioned in the original story; the latter category included mention of motives, feelings, or reactions that had not been mentioned in the story.

Table 2 shows the results of this analysis. As can be seen, the groups did not differ in the total number of intrusion errors made. However, the protocols indicated a clear contrast in the types of errors made by the neutral and arousal groups. The neutral group reconstructed plot segments twice as often as did the arousal group. In contrast, subjects in the arousal group were twice as likely

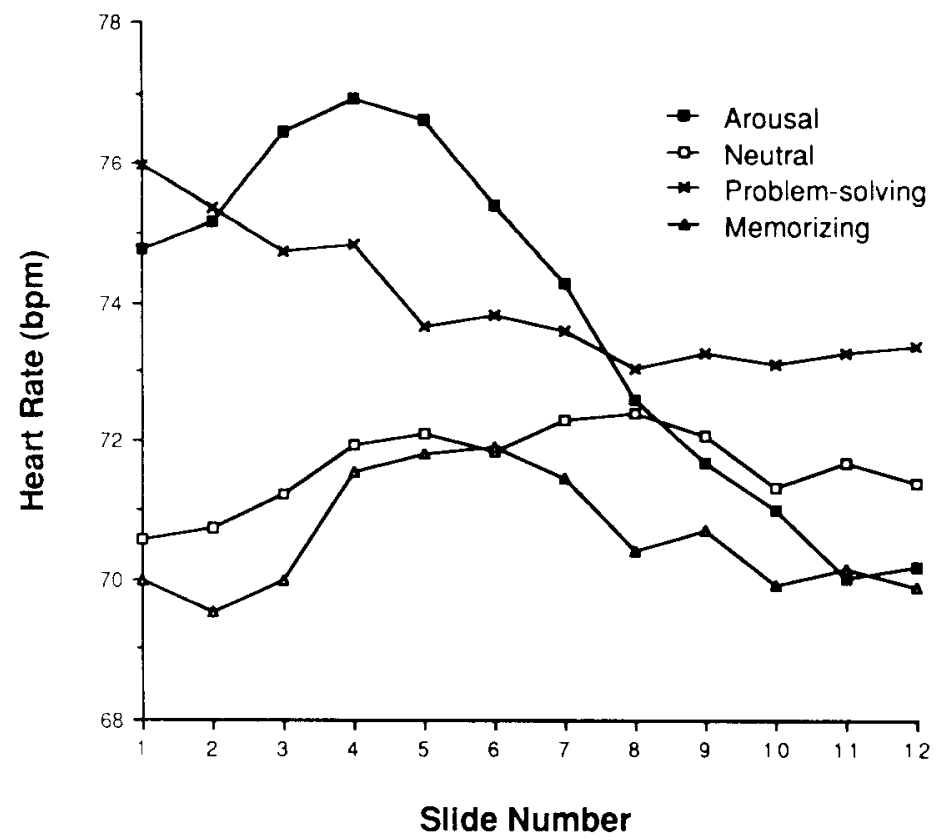

Figure 5. Slide-by-slide heart-rate values. 
Table 2

Types of Intrusion Errors in Neutral and Arousal Recall Protocols

\begin{tabular}{lcc} 
& \multicolumn{3}{c}{ Types of Intrusions } \\
\cline { 2 - 3 } Group & Plot & Character \\
\hline Arousal & 8 & 11 \\
Neutral & 17 & 5 \\
\hline
\end{tabular}

to embellish around these themes than were subjects of the neutral group. This contrast is statistically reliable $\left(\chi^{2}=5.31, p<.025\right)$.

\section{Memory for the Sentences}

Subjects did reasonably well in remembering the exact wording of the sentences originally heard. In the 4AFC procedure, chance performance would be $25 \%$. The subjects in the neutral group responded correctly for $52.5 \%$ of the sentences; the subjects in the arousal group responded correctly for $65.8 \%$. The memorizing and problem-solving groups yielded intermediate performance, at $58.8 \%$ and $58.9 \%$, respectively. An ANOVA did not show a main effect of group $[F(3,36)=2.031$, n.s.]. However, pairwise comparisons indicated that the neutral group's performance was reliably worse than that of the arousal group $[t(18)=2.642, p<.02]$. There was also a strong trend toward poorer performance in the neutral group than in either the memorizing or the problemsolving group $[t(18)=1.874$ and 1.994 , respectively, both $p s<.10]$. There were no reliable differences among the arousal, memorizing, and problem-solving groups.

\section{DISCUSSION}

According to these data, emotion appears to have had multiple effects on memory. First-and this is consistent with prior findings-arousal appears to have promoted memory for central information: In our recall data, this emerges as a statistically reliable advantage for the arousal group in both central information recalled and the number of slides recalled. In the recognition data, the arousal group outperforms all other groups, with its advantage most pronounced in memory for the middle phase of the procedure-that is, for the arousing materials themselves.

Second, and contrary to the Easterbrook or "weaponfocus" claim, according to these data arousal did not narrow the range of what got encoded, nor did it simply bias attention toward one sort of material or another. Instead, the emotional arousal seems actually to have enhanced the subjects' range of attention, and to have produced fuller, more detailed memory. This is visible (but not statistically reliable) in the recall data, and it is reflected more fully in the recognition data.

Third, arousal seems at the same time to have had a potentially deleterious effect on memory, leading subjects to overinterpret the memory at the time of recollection. This is visible in the pattern of intrusion errors.

Why do arousal subjects show an advantage in memory for peripheral information? Several plausible possibilities can be dismissed. First, one could attempt to argue that emotion influences how we deploy attention, perhaps drawing attention to peripheral and superfluous detail, away from more noxious elements at the event's core. However, the implied zero-sum process would lead us to expect poorer encoding of the event's central structure. Obviously, this is not what our results show. Instead, arousal subjects outperformed the other groups with both central and peripheral material. For the same reasons, we can reject accounts that assign arousal the role of narrowing the focus of attention. This narrowing of focus is, of course, the Easterbrook hypothesis described earlier, which predicted superior performance in the arousal group for either central or peripheral information, but not both-again, contrary to what we found.

Perhaps arousal increases subjects' rehearsal of the encoded information, rather than encoding or "pick-up" per se. If the arousal version of the story had been intrinsically more interesting than the neutral one, subjects might have continued to think about it after the presentation, creating a retention advantage. However, it is not clear why arousal subjects would have rehearsed peripheral details, and only details from the second half of the story. Recall, though, that the arousal group's advantage is most clearly visible for precisely these details. Moreover, the arousal advantage in peripheral memory is clearly not focused on the most interesting parts of the material (Slides 6-8), but rather spread throughout the full second half of the presentation. Last, and perhaps most persuasive, enhanced rehearsal would seem most likely in the memorizing group. Thus, if rehearsal underlies the arousal effect, one would expect comparable performance in these two groups. This was not the case; the memorizers matched the arousal group's recognition performance for central items, but not for peripheral ones. In fact, the memorizers showed exactly the disadvantage with peripheral detail that we would expect if rehearsal concentrated on plot. (See, e.g., Figure 4, especially the memorizing group's performance for the second half of the presentation.)

Finally, perhaps subjects have a greater repertoire of story schemata for emotional materials, and, within these, they can more readily assimilate (and remember) emotional events they witness or experience. This would be fully consistent with the intrusions observed in recall. However, the schemata account would still leave unexplained the peripheral memory. An available schematic frame would surely promote memory for plot and central materials, but it would not be expected to aid memory for minutiae of appearance and the like.

The results therefore do seem to indicate a genuine encoding boost for peripheral information in the arousal group. Arousal subjects were not merely reporting more (especially given the forced-choice procedure); instead, they seem somehow to have encoded more. We can think of two ways this may have come about. One is that arousal subjects may simply have more attention (capacity, resources) to deploy, thus aiding performance overall (see 
Kahneman, 1973). Alternatively, there is some evidence that arousal may directly and specifically facilitate encoding. Gold (1987), for example, has proposed that adrenaline promotes memory encoding by raising plasma glucose levels (although the mechanism by which the glucose affects memory is not yet clear). Interestingly, this would return us to a proposal made by Livingston (1967) and echoed by Brown and Kulik (1977) in their classic paper on flashbulb memories. These authors proposed a special encoding mechanism (a "Now print!' mechanism), triggered by a mix of surprise and perceived consequentiality of an event. Brown and Kulik acknowledged that consequentiality might serve as an index (or cause) of emotionality and arousal, thus anticipating the present proposal. ${ }^{4}$

Finally, we believe that, to the various effects of emotional arousal we have mentioned so far, one more effect must be added. A number of studies indicate that, whereas emotional arousal promotes long-term retention, arousal works against short-term remembering (Kleinsmith \& Kaplan, 1963). While the relevant data for this point come mostly from memory for words and the like, we believe that more complex materials-and memory for peripheral detail-may follow the same pattern. ${ }^{5}$

This point is directly pertinent to the seeming contrast between our data and the standard findings in Easterbrook studies. Most of the data relevant to the Easterbrook claim look at arousal's impact on the on-line utilization of cues. Arousal does indeed seem to diminish the range of cues that has been used in these studies, leading us to argue that these studies reflect arousal's deleterious impact on short-term retention. The most natural account of these data (given the present results) is that emotional arousal simultaneously facilitates encoding and disrupts retrieval (and use) of the retrieved information. In the Easterbrook studies, and in short-term retention, the latter effect masks the former. With long-term retention, the emotion is not present at the time of retrieval, and so the latter effect is irrelevant. To put this differently, the notion of a weapon-focus may be ambiguous: What the eyewitness will remember immediately after the crime may be only the weapon; what the eyewitness might later remember may be far broader. (Unfortunately, testimony, based on the earlier and narrower recollection, may heavily influence the later report!)

In summary, then, we know from prior evidence that emotional arousal triggers vivid recollection. The current study indicates that emotional arousal in fact triggers vivid and accurate recollection. This is not to say that emotion's impact on memory is simple or uniformly beneficial. We have seen indications that emotionality encourages a certain species of intrusion error; we have also suggested that emotionality may impede the retrieval and use of remembered information.

It remains to be seen whether we have catalogued the full range of emotion's contributions to memory. For example, will similar results be obtained with different levels of arousal (specifically, levels higher than those easily achieved in the laboratory)? Likewise, we leave open the possibility of different results with different species of arousal (but see Reisberg et al., 1988). That is, our stimuli evoked the species of arousal associated with an orienting response, rather than the arousal associated with flight (including heart-rate acceleration). It remains to be demonstrated that the effects of these two are comparable.

Thus we close on a cautious note. A central theme in this discussion has been the diversity of emotion's effects on memory, and we take that theme to heart in interpreting our own results. Emotion's impacts on cognition are far from well understood. Thus, the generalizability of our claims is a matter to be settled empirically, as is the question of what other consequences arousal might have for memory. It is becoming increasingly clear that emotions' impacts on memory are many and diverse. No simple claims, and no single study, seems likely to encompass the full range of these.

\section{REFERENCES}

BARTLETT. F. C. (1932). Remembering: A study in experimental and social psychology. Cambridge, England: Cambridge University Press. Bohlin, G., \& Graham, F. K. (1977). Cardiac deceleration and reflex blink facilitation. Psychophysiology, 14, 423-430.

Brown, R., \&ulik, J. (1977). Flashbulb memories. Cognition, 5 , 73-99.

Bruner, J., Matter, J., \& Papanek, M. (1955). Breadth of learning as a function of drive level and mechanization. Psychological Review, 42, $1-10$.

Christianson, S.-A. (1984). The relationship between induced emotional arousal and amnesia. Scandinavian Joumal of Psychology, 25, 147-160.

Christianson, S.-A., \& Loftus, E. F. (1987). Memory for traumatic events. Applied Cognitive Psychology, 1, 225-239.

Christianson, S.-A., Loftus, E. F., \& Nilsson, L. (1987, November). Memory for emotional events. Paper presented at the annual meeting of the Psychonomic Society, Seattle, WA.

Clark, M. S., Milberg, S., Ross, J. (1983). Arousal cues arousalrelated material in memory: Implications for understanding effects of mood on memory. Journal of Verbal Learning \& Verbal Behavior, 22, 633-649.

EASTERBROOK, J. (1959). The effect of emotion on cue utilization and the organization of behavior. Psychological Review, 66, 183-201.

EYSENCK, M. (1982). Attention and arousal: Cognition and performance. Berlin: Springer-Verlag.

Gold, P. (1987). Sweet memories. American Scientist, 75, 151-155.

Graham, F. K. (1979). Distinguishing among orienting, defense and startle reflexes. In H. D. Kimmel, E. H. van Olst, \& J. G. Orlebeke (Eds.), The orienting reflex in humans (pp. 137-167). Hillsdale, NJ: Eribaum.

Kahneman, D. (1973). Attention and effort. Englewood Cliffs, NJ: Prentice Hall.

Kleinsmith, L., \& KaPlan, S. (1963). Paired associate learning as a function of arousal and interpolated interval. Joumal of Experimental Psychology, 65, 190-193.

LACEY, J., \& LACEY, B. (1974). On heart rate responses and behavior: A reply to Elliott. Journal of Personality \& Social Psychology, 30, $1-18$.

LIVINGSTON, R. (1967). Brain circuitry relating to complex behavior. In G. Quarton, T. Melnechuck, \& F. Schmitt (Eds.), The neurosciences: A study program (pp. 499-514). New York: Rockefeller University Press

Loftus, E. F. (1979). Eyewitness testimony. Cambridge, MA: Harvard University Press

LofTus, E. F. (1982). Remembering recent experiences. In L. Cer- 
mak (Ed.), Human memory and amnesia (pp. 239-255). Hillsdale, NJ: Erlbaum.

LofTus, E. F., \& BURNS, T. (1982). Mental shock can produce retrograde amnesia. Memory \& Cognition, 10, 318-323.

Mandler, G. (1975). Mind and emotion. New York: Wiley.

Marks, D. (1972). Individual differences in the vividness of visual imagery and their effect on function. In P. Sheehan (Ed.), The function and nature of imagery (pp. 83-108). New York: Academic Press.

McCloskey, M., \& EGETH, H. (1983). Eyewitness identification: What can a psychologist tell a jury? American Psychologist, 38, 550-563.

McCloskey, M., WiBle, C., \& Cohen, N. (1988). Is there a special flashbulb-memory mechanism? Journal of Experimental Psychology: General, 117, 171-181.

Mehrabian, A. (1977). Individual differences in stimulus screening and arousability. Journal of Personality, 45, 237-250.

MinSKY, M. (1975). A framework for representing knowledge. In P. H. Winston (Ed.), The psychology of computer vision (pp. 211-277). New York: McGraw-Hill.

NEISSER, U. (ED.) (1982). Memory observed: Remembering in natural contexts. San Francisco: W. H. Freeman.

NeIsser, U. (1986). Remembering Pearl Harbor: Reply to Thompson and Cowan. Cognition, 23, 285-286.

Pillemer, D. (1984). Flashbulb memories of the assassination attempt on President Reagan. Cognition, 16, 63-80.

Reisberg, D., Heuer, F., Mclean, J., \& O'Shaughnessy, M. (1988). The quantity, not the quality, of affect predicts memory vividness. Bulletin of the Psychonomic Society, 26, 100-103.

Rosch, E. (1978). Principles of categorization. In E. Rosch \& B. B. Lloyd (Eds.), Cognition and categorization (pp. 27-48). Hillsdale, NJ: Erlbaum.

Rubin, D., \& Kozin, M. (1984). Vivid memories. Cognition, 16, 81-95. RuBin, D. (1986). Autobiographical memory. Cambridge, England: Cambridge University Press.

Thompson, C., \& Cowan, T. (1986). Flashbulb memories: A nicer interpretation of a Neisser recollection. Cognition, 22, 199-200.

\section{NOTES}

1. The present study will allow us to address the impact of this alleged selective set empirically. Note in particular the contrast between our results with the arousal and memorizing or problem-solving groups.

2. Because of inaudibility of the tapes, two protocols (one from the neutral, one from the problem-solving group) could not be transcribed. The degrees of freedom in the tests have therefore been reduced. In addition, given the unequal $N \mathrm{~s}$ in the four cells, we report only pairwise comparisons among the cells. Note also that the recall data (unlike the recognition data) could not be analyzed by phase. In the recall protocols, it was often impossible to tell the "source" of recalled information. If a subject recalled some particular detail, that subject might have been recalling the detail from materials viewed in any of the three phases. This precluded analyses of the recall data by phase.

3. The main contrasts of interest here concern the comparisons between the neutral and arousal groups. Given this, and given the laborious nature of the analyses reported in this section, we will report data only from the arousal and neutral groups.

4. We do not, however, wish to endorse Brown and Kulik's (1977) proposal that flashbulb memories constitute a class of memory sui generis; our suggestion instead is that arousal's effects may be similar in nature to those proposed by Brown and Kulik, but broader in application. For further discussion of whether flashbulbs constitute a unique category of memory, see McCloskey et al. (1988). Note that the McCloskey et al. data make clear that flashbulb memories of emotional events are not uniformly accurate. This in no way conflicts with the present findings, which show only that arousal promotes memory accuracy; it does not guarantee accuracy.

5. Some support for this suggestion comes from Christianson (1984). As we mentioned earlier, Christianson obtains in some cells but not others the crossover interaction reported by Kleinsmith and Kaplan (1963). Christianson does report a statistically reliable interaction in the appropriate direction-with the disadvantage for arousal clear in initial testing, and much reduced in later testing.

Appendix

Sentences accompanying the slide presentation

\begin{tabular}{|c|c|}
\hline Neutral version & Arousal version \\
\hline $\begin{array}{l}\text { 1. Mother and son are leaving home in the } \\
\text { morning. }\end{array}$ & $\begin{array}{l}\text { Mother and son are leaving home in the } \\
\text { morning. }\end{array}$ \\
\hline 2. They make sure that crossing Park Road is safe. & They make sure that crossing Park Road is safe. \\
\hline 3. She is taking him to visit his Dad at work. & She is taking him to visit his Dad at work. \\
\hline 4. Father is head mechanic at the nearby garage. & Father is chief surgeon at the nearby hospital. \\
\hline 5. Earlier in the day this car had to be towed in. & Earlier in the day there was a terrible accident. \\
\hline $\begin{array}{l}\text { 6. The garage crew has been trying to locate the } \\
\text { problem. }\end{array}$ & $\begin{array}{l}\text { The surgical team has been fighting to save the } \\
\text { victims. }\end{array}$ \\
\hline 7. Father was able to find the broken connection. & Father was able to restore the severed limbs. \\
\hline 8. He is pleased that his son watched the repair. & He is pleased that his \\
\hline 9. Mother leaves the garage being late for her job & $\begin{array}{l}\text { Mother leaves the hospital upset by what she } \\
\text { saw. }\end{array}$ \\
\hline 10. Heading $t$ & Heading to call work she passes a police station. \\
\hline & Mother asks her boss to get the day off. \\
\hline $\begin{array}{l}\text { 12. She tries to hail a cab downtown at the } \\
\text { number } 3 \text { bus stop. }\end{array}$ & $\begin{array}{l}\text { She tries to hail a cab home at the } \\
\text { number } 3 \text { bus stop. }\end{array}$ \\
\hline
\end{tabular}

(Manuscript received July 1988 ;

revision accepted for publication January 5, 1990.) 